\title{
A simulation study of galactic proton modulation from solar minimum to maximum conditions
}

\section{D.C. Ndiitwani, ${ }^{a, b, *}$ M.D. Ngobeni, ${ }^{a, b}$ O.P.M. Aslam, ${ }^{a}$ D. Bisschoff, ${ }^{a}$ M.S. Potgieter, ${ }^{c}$ M. Boezio, $^{d}$ R. Munini ${ }^{d}$ and V.V. Mikhailov ${ }^{e}$}

${ }^{a}$ Centre for Space Research, North-West University, Potchefstroom, South Africa

${ }^{b}$ School of Physical and Chemical Sciences, North-West University, Mmabatho, South Africa

${ }^{c}$ Institute for Experimental and Applied Physics, CA University in Kiel, Germany

${ }^{d}$ INFN, Sezione di Trieste, Italy

${ }^{e}$ National Research Nuclear University MEPHI, Moscow, Russia

E-mail: Dzivhuluwani.Ndiitwani@nwu.ac.za

\begin{abstract}
The observation of various cosmic ray particles at the Earth had been done with the PAMELA space detector for almost 10 years, from June 2006 to January 2016. The AMS-02 space experiment provides similar cosmic ray data. The purpose of this work is to utilize the available state-of-theart numerical modulation model for the transport of cosmic rays in the heliosphere to compute the modulation of galactic protons from minimum to maximum solar activity. These modeling results, which simulate realistic heliospheric conditions, are compared to proton observations from PAMELA taken between 2006 and 2010 and to similar AMS-02 observations after 2011. It will be shown how differently modulation mechanisms influence the time-evolution of the proton spectra when modulation conditions change from minimum to maximum, including a reversal of the polarity of the heliospheric magnetic field.
\end{abstract}

$37^{\text {th }}$ International Cosmic Ray Conference (ICRC 2021)

July 12th - 23rd, 2021

Online - Berlin, Germany

\footnotetext{
*Presenter
} 


\section{Introduction}

The influence of solar activity on the modulation of galactic cosmic rays (GCRs) in conjuction with the observation of the unusual solar minimum between solar cycles 23 and 24 has increased interest in the study of the modulation of GCRs in the heliosphere. Solar cycles 23-24 differed from previously recorded solar minimum periods so that the highest levels of GCRs since the beginning of space exploration were recorded at Earth in late 2009 [1-3]. This period was characterised by a much weaker heliospheric magnetic field (HMF) and by tilt angles of the heliospheric current sheet (HCS) not decreasing as rapidly as the magnitude of HMF at Earth, reaching a minimum value at the end of 2009. This led to the insight that modulation conditions in the heliosphere had reached unprecedented quiet levels. This stirred up a series of modulation modelling studies in order to establish what adjustment need to be made as required in mathematical and numerical models with respect to the elements of the diffusion and drift tensor in the relevant transport equation in order to reproduce GCR observations at Earth. In this context, $[4,5]$ modelled proton, electron and positron spectral features observed by PAMELA, whereas [6] studied the modelling of GCR protons and helium nuclei as observed by PAMELA between 2006 and 2009 in a very quiet heliosphere, and [7] modelled also proton and anti-proton observations obtained after 2011.

This work extends on this approach and studies the modulation of galactic protons from 2006 until 2017, a period coinciding with solar activity changing from minimum to maximum conditions, including a HMF polarity reversal. This study takes advantage of simultaneous and continuous observations of galactic protons from the PAMELA [10] and AMS02 [11] space experiments to achieve the objective of the study. Both [7,8] successfully computed time and charge-sign dependent modulation for the period that covers the previous long and unusual deep solar minimum activity and the recent maximum period. The mentioned studies investigated how the main modulation processes including particle drifts and the major diffusion coefficients had evolved during the period investigated and subsequently how the corresponding charge-sign dependent modulation had occurred. A report on how modulation parameters had to change in modelling of previous solar cycles, particularly charge-sign dependence along the Ulysses trajectory, was made by [12].

In this paper a 3D numerical model, including all four major modulation processes, is applied to compute galactic protons as observed by PAMELA from 2006 to 2009 and to AMS02 for period from 2011 up to 2017. The modelling parameters which successfully reproduced proton spectra for the previous solar minimum period is adopted for the current study and a similar approach as mentioned above has been used to establish modulation parameters to reproduce proton spectra observed by AMS02 for the period 2011 up to 2017.

\section{Numerical model and modulation parameters}

The three-dimensional (3D) steady-state modulation model as described by [4], and recently improved by [8], is used to calculate proton spectra for monthly (and 27-day averages) up to sixmonth averages depending on the GCRs being studied and the particular application. The model is based on the numerical solution of Parker's [13] transport equation (TPE):

$$
-\left(\mathbf{V}+\left\langle\mathbf{v}_{D}\right\rangle\right) \cdot \nabla f+\nabla \cdot\left(\mathbf{K}_{s} \cdot \nabla f\right)+\frac{1}{3}(\nabla \cdot \mathbf{V}) \frac{\partial f}{\partial \ln P}+J_{\text {source }}=0
$$


where $f(\mathbf{r}, P)$ is the cosmic ray distribution function; $P$ is rigidity, $\mathbf{r}$ is position, and $\mathbf{V}$ is the solar wind velocity. The terms from left to right represent convection caused by expanding solar wind, gradient, curvature and HCS drifts, diffusion, adiabatic energy changes and a source function (in our case equal to zero), respectively. The diffusion tensor $\mathbf{K}_{\mathbf{s}}$ consists of a parallel diffusion coefficient, $K_{\|}$, and two perpendicular diffusion coefficients in the radial direction $K_{\perp r}$ and in the polar direction $K_{\perp \theta}$. The modulation boundary is assumed to be the heliopause (HP) specified in the model at $122 \mathrm{AU}$ where the proton very LIS is used as initial spectrum which is then modulated from the HP up to the Earth. The study adopts the proton very LIS originally from [4] which was later updated by [14].

For our modelling to calculate proton spectra (differential intensities), the general expression for $K_{\|}$ to the average background HMF, with magnitude $B$, is given by:

$$
K_{\|}=\left(K_{\|}\right)_{0} \beta\left(\frac{B_{0}}{B}\right)\left(\frac{P}{P_{0}}\right)^{c_{1}}\left[\frac{\left(\frac{P}{P_{o}}\right)^{c_{3}}+\left(\frac{P_{k}}{P_{o}}\right)^{c_{3}}}{1+\left(\frac{P_{k}}{P_{o}}\right)^{c_{3}}}\right]^{\frac{c_{2 \|} c_{1}}{c_{3}}},
$$

where $\left(K_{\|}\right)_{0}$ is a scaling parameter in units of $10^{22} \mathrm{~cm}^{2} \mathrm{~s}^{-1}$, with $P_{0}=1.0 \mathrm{GV}$ and $B_{0}=1.0 \mathrm{nT}$. The constants $c$ as power indices, provide for two power laws and $P_{k}$ specifies the rigidity at which the transition between the two power laws occurs. In this case, $c_{1}$ is a power index that changes with time and $c_{2 \|}$, together with $c_{1}$, determine the rigidity dependent slope above and below a rigidity with value $P_{k}$, whereas $c_{3}$ determines the smoothness of the transition. The values of these constants that were used to successfully compute proton spectra are listed by [5] for the years 2006 to 2009. In this study a similar exercise extended for the period 2011 to 2017 has been performed to establish these power indices for $K_{\|}$to reproduce spectra compatible to AMS02 observations. The rigidity dependence of $K_{\|}$, indicating the two power-law slopes, at Earth is illustrated in Figure 1 as the corresponding parallel mean free paths (MFP; $\lambda_{\|}$) for four selected times; with $K=\lambda(v / 3$ ) where $v$ is the particle speed and $\beta=v / c$, with $c$ the speed of light. How $K_{\|}$relates to $K_{\perp r}$ and $K_{\perp \theta}$ has been described in detail by [6,8] and is not repeated here; see also [9].

The model requires the determination of 27-day averaged values for all modulation parameters that vary with time and as such with solar activity e.g. the tilt angle $\alpha$ of the HCS and magnitude $B$ of the HMF at Earth. Figure 2 shows the variations of $\alpha$ and $B$ at Earth from 2005 to June 2017. These obtained values are read into the numerical modelling code as input parameters to simulate appropriated modulation conditions. Also shown, as red circle dots, are the calculated 15 monthmoving-averages for $\alpha$ and $B$. These calculated averages establish realistic heliospheric conditions as they are propagated into the heliosphere with the solar wind, which latitudinal dependence is also changing from solar minimum to maximum. See $[5,6,8]$ for an elaborate discussion of the details of this approach as applied to GCR data sets observed by PAMELA (e.g. [10, 17]) and for AMS02 (e.g. [11, 16]).

\section{Simulated proton spectra}

Aslam et al. [5] successfully reproduced the 6 month-averaged PAMELA positron spectra from July 2006 to December 2009 by carefully adjusting the diffusion and drift coefficients. These spectra were calculated for six month periods (meaning the first and last six months of every year 


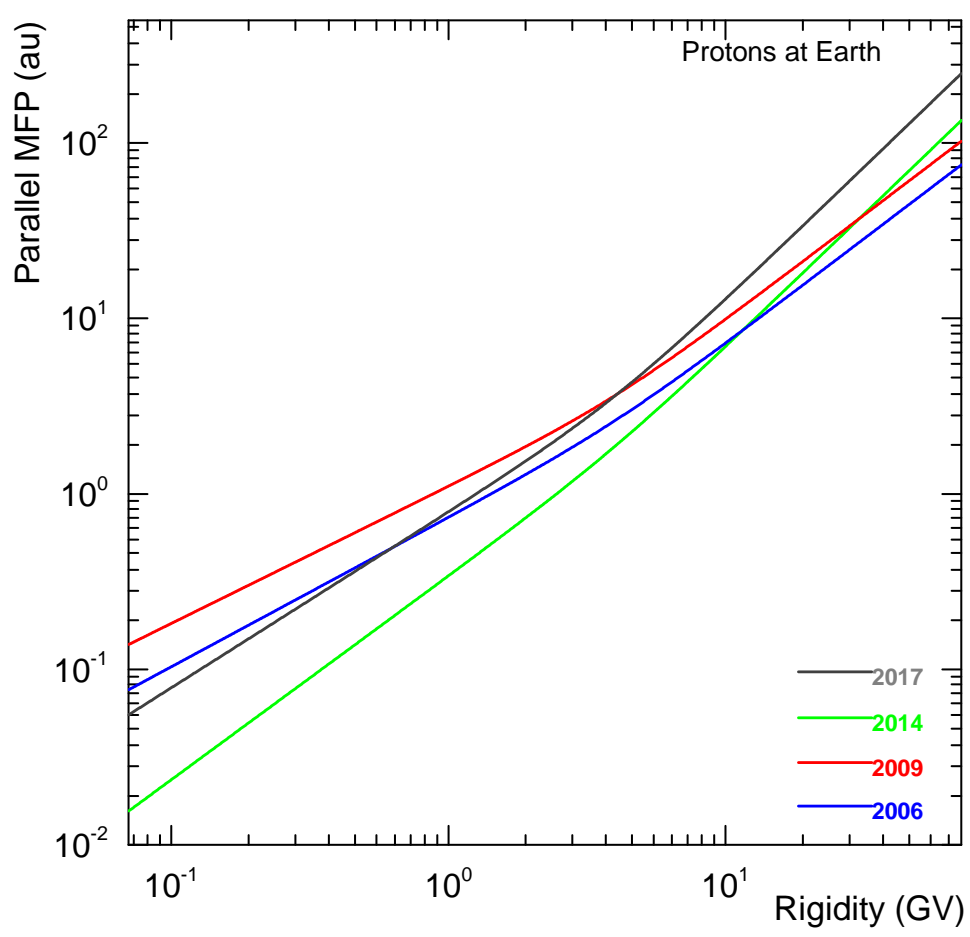

Figure 1: Rigidity dependence of the proton parallel mean free path (MFP, $\lambda_{\|}$) in units of au, that are required to reproduce the four representative spectra as shown in Figure 3

mainly because of experimental constraints) using the calculated average values of $\alpha$ and $B$. In this study a similar approach is used for proton spectra from 2006 to 2017, but for protons shorter averages can be selected, if required, because of better experimental statistics which allow proton spectra to be published for each Carrington or Bartels rotation and then used to calculate longer averages as required such as for every 6 months. Figure 3 shows computed proton spectra for selected periods and overlaid by the observed spectra at Earth with respect to the corresponding very LIS. The spectra shown are not the only ones calculated for this study but shown here as representative spectra selected for distinguishable periods of solar activity. Comparing these solutions with observations demonstrates that the model can indeed reproduce both PAMELA and AMS02 proton spectra very well across all rigidities and from minimum to maximum solar activity. The model also simulates observed spectral features such as softening and hardening of the spectra as solar activity decreases or increases.

To achieve what is mentioned above, a different rigidity dependence had to be assumed, as shown in the first figure above, by changing the value of $\left(K_{\|}\right)_{0}$ and $c_{1}$ in Eq. 1 with time as shown in Table 1 for the years from 2011 to 2017; here indicated in six months intervals. The values of $c_{2 \|}$ and $P_{k}$ were kept the same through out the period at 1.52 and $4.0 \mathrm{GV}$, respectively. The preceding period, before 2011, had been studied previously and reported in detail by $[4,5,15]$. The explicit values of $c_{1}$ and $\left(K_{\|}\right)_{0}$ as listed, convey how the rigidity dependence of $K_{\|}$needs to change with time as shown in Figure 1 to reproduce proton spectra as shown in Figure 3 and for other intermediate 


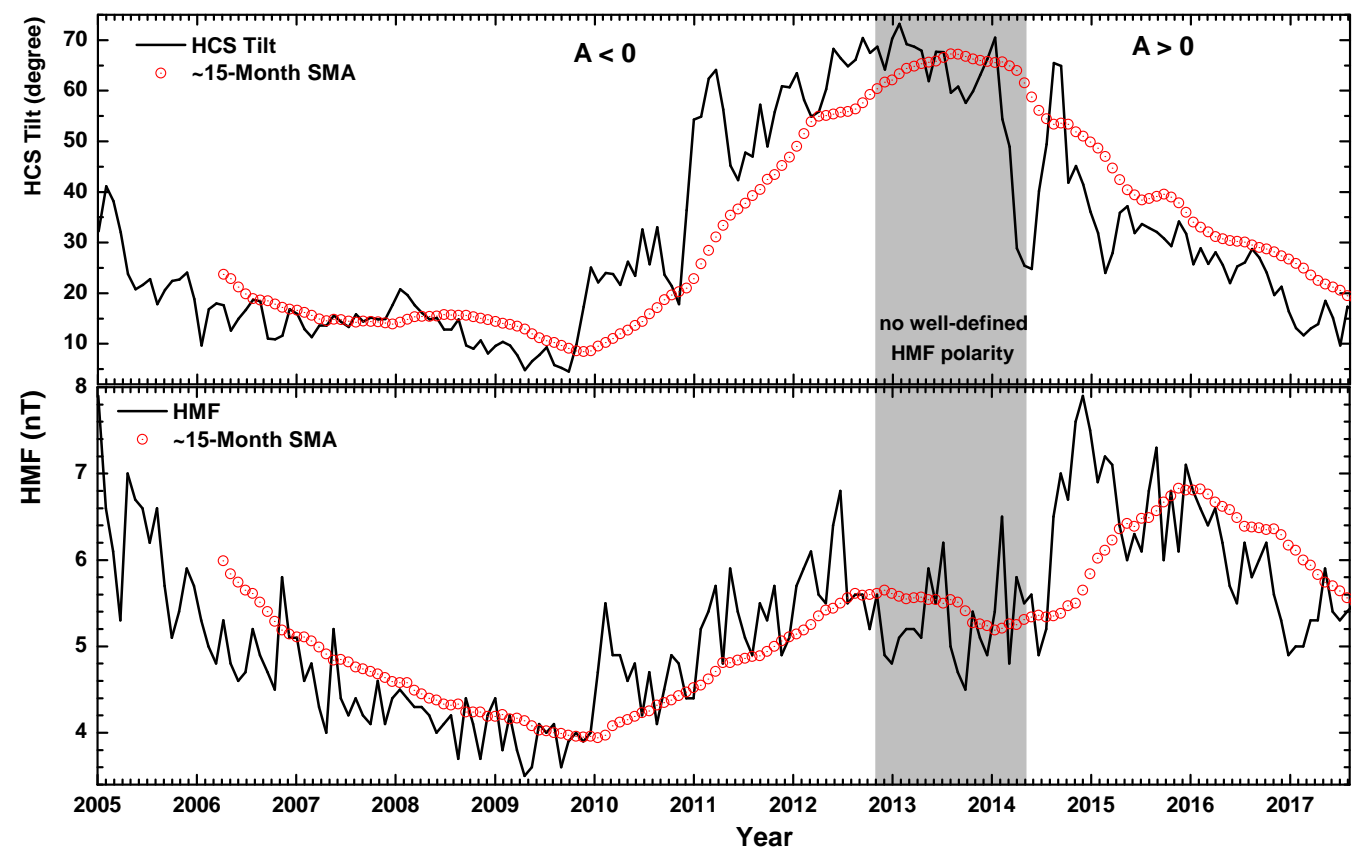

Figure 2: Top panel shows the tilt angle $\alpha$ of the HCS (black line) at the Earth from January 2005 to June 2017 taken from http://wso.stanford.edu along with 17 Carrington rotation (15 months) moving averages (red circle dots). Bottom panel shows the magnitude of the HMF at the Earth (black line) for the same period taken from http://omniweb.gsfc.nasa.gov, along with 17 Bartels (15 months) moving averages (red circle dots). Shaded portions indicate the estimated period of the polarity reversal of the HMF which is considered a period of no well-defined HMF polarity.

spectra. The values in Table 1, together with those reported by [5], indicate how the physics of solar modulation as related to parallel diffusion needs to change over time from the solar minimum of 2006-2009 to the period of increasing solar activity from and after 2010, reaching solar maximum in 2015, including the HMF polarity reversal phase from November 2012 to March 2014.

\begin{tabular}{cccccccc}
\hline \hline Parameter & $2011 \mathrm{a}$ & $2012 \mathrm{a}$ & $2013 \mathrm{a}$ & $2014 \mathrm{a}$ & $2015 \mathrm{a}$ & $2016 \mathrm{a}$ & $2017 \mathrm{a}$ \\
\hline $\mathrm{CR}$ & 2426 & 2440 & 2453 & 2467 & 2480 & 2494 & 2506 \\
\hline$c_{1}$ & 0.81 & 0.90 & 1.01 & 1.16 & 1.25 & 1.25 & 1.0 \\
$\left(K_{\|}\right)_{0}$ & 51.443 & 45.743 & 33.021 & 27.460 & 30.240 & 50.262 & 68.128 \\
\hline \hline Parameter & $2011 \mathrm{~b}$ & $2012 \mathrm{~b}$ & $2013 \mathrm{~b}$ & $2014 \mathrm{~b}$ & $2015 \mathrm{~b}$ & $2016 \mathrm{~b}$ & \\
\hline$c_{1}$ & 0.87 & 1.01 & 1.14 & 1.10 & 1.28 & 1.14 & \\
$\left(K_{\|}\right)_{0}$ & 49.150 & 38.166 & 26.764 & 32.326 & 41.363 & 64.304 & \\
\hline \hline
\end{tabular}

Table 1: Power indices and constants used in Eq. 1 to reproduce the observed proton spectra for every six-months period of each year, from 2011a to 2017a; CR indicates Carrington rotation.

Recent modeling studies based on a similar 3D model and complimentary to ours, are reported by $[9,18-20]$. 


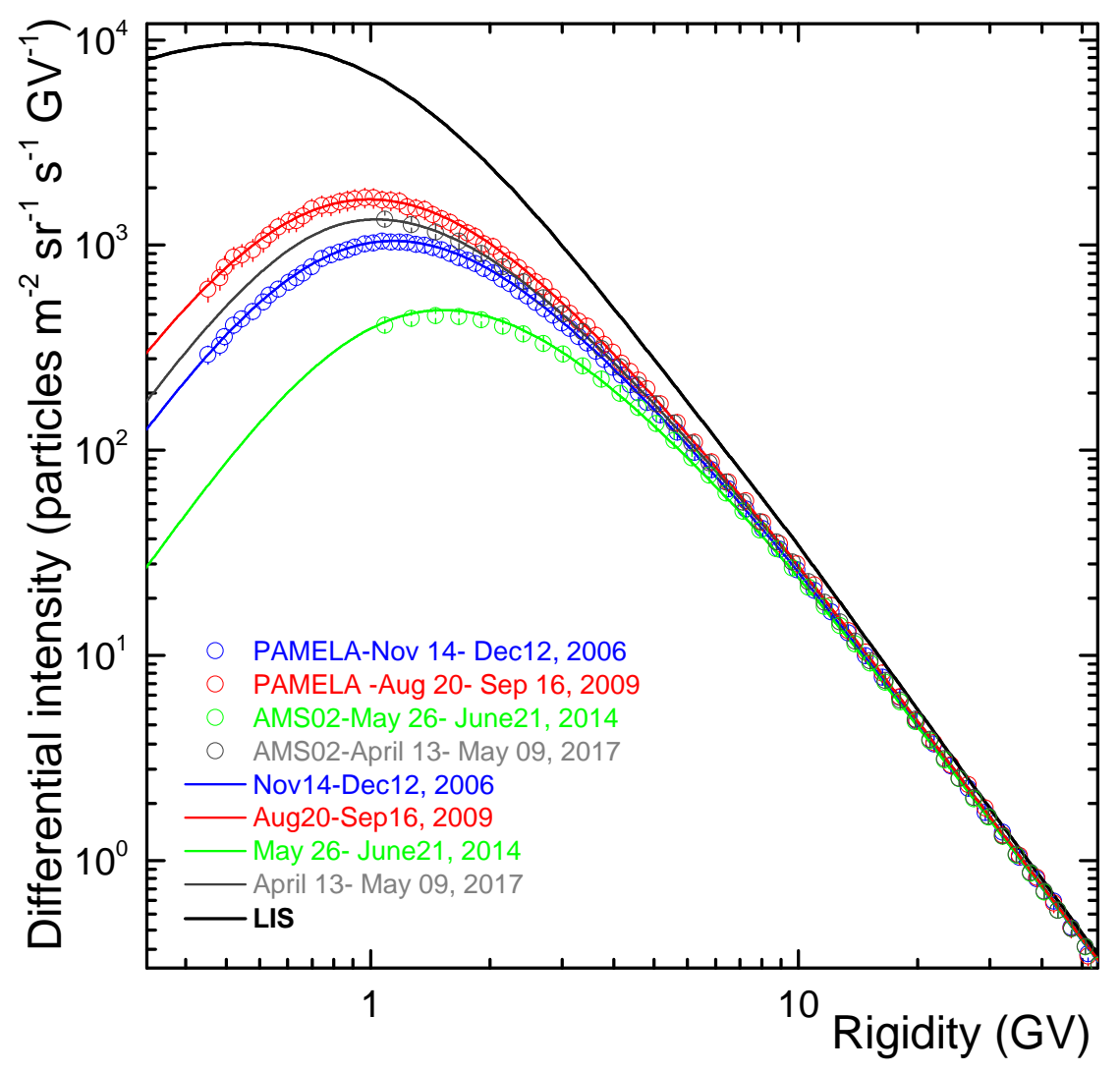

Figure 3: Modulated proton spectra (coloured lines) computed with respect to the very LIS at 122 au for protons (upper black solid line) during periods of different solar activity for selected times as indicated, and compared to PAMELA and AMS02 observations at Earth as indicated by open coloured circles.

\section{Summary and Conclusion}

This work presents a preliminary study and abridged report of simulating proton spectra from solar minimum to maximum conditions, including a reversal of the HMF polarity. To accomplish this objective a comprehensive 3D numerical model has been applied to compute proton spectra that in all respects are compatible with PAMELA and AMS02 observations. It follows from this comparison of simulated spectra with precise observations how the rigidity dependence of the parallel diffusion coefficient for protons needs to change with solar activity to reproduce observations from 2006 to 2017.

\section{Acknowledgement}

DCN and MDN thank the SA National Research Foundation (NRF) for partial financial support under the Joint Science and Technology Research Collaboration between SA and Russia (Grant no: 118915). DCN also acknowledges that the opinions, findings and conclusions or recommendations expressed in any publication generated by the NRF supported research is that of the authors alone, and that the NRF accepts no liability whatsoever in this regard. DB and OPMA acknowledge 
the financial support from the NWU post-doctoral programme. VVM acknowledges the financial support from the Russia Foundation for Basic Research (RFBR) according to research project no.1952-60003. M.B and RM acknowledges partial financial support from the INFN Grant "giovani", project ASMDM.

\section{References}

[1] O.P.M. Aslam and Badruddin, SoPh 269, 279, 2012.

[2] M.S. Potgieter et al., ApJ. 141, 810, 2015.

[3] M.S. Potgieter, Adv. Space Res. 60, 848, 2017.

[4] E.E. Vos and M.S. Potgieter, ApJ. 119, 815, 2015.

[5] O.P.M. Aslam et al., ApJ. 70, 873, 2019.

[6] M.D. Ngobeni et al., Astrophy. Space Sci. 182, 365, 2020.

[7] O.P.M. Aslam et al., PoS (ICRC2019), 358, 1053, 2019.

[8] O.P.M. Aslam et al., ApJ. 215, 909, 2021.

[9] M.D. Ngobeni et al., PoS (ICRC2021), 264, 2021.

[10] O. Adriani, et al., PhR, 323, 544, 2014.

[11] M. Aguilar et al., PhRVL, 141102, 110, 2013.

[12] D.C. Ndiitwani, et al., Ann. Geophys. 23, 1, 2005.

[13] E.N. Parker, Planet. Space Sci. 13, 9. 1965.

[14] D. Bisschoff et al., ApJ. 878, 592019.

[15] E.E. Vos and M.S. Potgieter, Solar Phys. 2181, 291, 2016.

[16] C. Corti et al., ApJ. 153, 871, 2019.

[17] M. Boezio et al., PoS (ICRC2017), 35, 1091, 2017.

[18] M.D. Ngobeni et al., PoS (ICRC2021), 262, 2021.

[19] M. Krainev et al., PoS (ICRC2021), 352, 2021.

[20] R. Munini et al., PoS (ICRC2021), 1128, 2021. 